UT-Komaba 00-10

\title{
Lattice QCD with the Overlap Fermions at Strong Gauge Coupling (II)
}

\author{
Ikuo Ichinosetand Keiichi Nagaő \\ Institute of Physics, University of Tokyo, Komaba, Tokyo, 153-8902 Japan
}

\begin{abstract}
In the previous paper we developed a strong-coupling expansion for the lattice QCD with the overlap fermions and showed that Lüscher's "extended" chiral symmetry is spontaneously broken in some parameter region of the overlap fermions. In this paper, we derive a low-energy effective action of hadrons and show that there exist quasi-Nambu-Goldstone bosons which are identified as the pions. The pion field is a nonlocal composite field of quark and anti-quark even at the strong-coupling limit because of the nonlocality of the overlap fermion formalism and Lüscher's chiral symmetry. The pions become massless in the limit of the vanishing bare-quark mass as it is desired. We furthermore examine symmetries of the overlap fermions with the hopping expansion and argue that there appear no other massless modes other than the pions.
\end{abstract}

\footnotetext{
${ }^{1}$ e-mail address: ikuo@hep1.c.u-tokyo.ac.jp

${ }^{2}$ e-mail address: nagao@hep1.c.u-tokyo.ac.jp
} 


\section{Introduction}

One of the long standing problems in the lattice gauge theory is the formulation of lattice fermions. Recently a very promising formulation named overlap fermion was proposed by Narayanan and Neuberger [1, 2] and it has been studied intensively by both analytic and numerical methods. In the previous paper [3] (which we shall refer to paper I hereafter), we slightly extended the overlap fermion by introducing a "hopping" parameter $t$ and studied the lattice QCD by using both the $t$-expansion and the strong-coupling expansion. There we calculated the effective potential of the chiral condensation and showed that Lüscher's extended chiral symmetry [⿴囗十 is spontaneously broken at certain parameter region of the overlap fermion. In this paper we shall derive an effective action of low-energy excitations and show that there exist quasi-Nambu-Goldstone bosons which are identified as the pions. As we show, the pion field is a nonlocal composite field of quark and anti-quark even at the strong-coupling limit.

This paper is organized as follows. In Sect.2, we shall give a brief review of the overlap fermion and its hopping expansion. We introduce nonlocal composite operators of quarks which transform "covariantly" under Lüscher's chiral transformation. In Sect.3, we derive the effective action of mesons by integrating out the gauge fields and the quarks. Lüscher's symmetry is spontaneously broken at strong gauge coupling as we showed in paper I. From the effective action, we show that there appear quasimassless mesons which are identified as the pions. In Sect.4, we discuss symmtries of the overlap fermion and their relation to the species doubling. We argue that there are no massless modes other than the pions. Physical meaning of the hopping expansion is also discussed. Section 5 is devoted for discussion and conclusion. 


\section{Hopping expansion}

Action of the overlap fermion on the $d$-dimensional lattice is given as follows,

$$
S_{F}=a^{d} \sum_{n, m} \bar{\psi}(m) D(m, n) \psi(n)
$$

where the covariant derivative $D(m, n)$ is defined as

$$
\begin{aligned}
D & =\frac{1}{a}\left(1+X \frac{1}{\sqrt{X^{\dagger} X}}\right) \\
X_{m n} & =\gamma_{\mu} C_{\mu}(t ; m, n)+B(t ; m, n), \\
C_{\mu}(t ; m, n) & =\frac{t}{2 a}\left[\delta_{m+\mu, n} U_{\mu}(m)-\delta_{m, n+\mu} U_{\mu}^{\dagger}(n)\right], \\
B(t ; m, n) & =-\frac{M_{0}}{a}+\frac{r}{2 a} \sum_{\mu}\left[2 \delta_{n, m}-t \delta_{m+\mu, n} U_{\mu}(m)-t \delta_{m, n+\mu} U_{\mu}^{\dagger}(n)\right],
\end{aligned}
$$

where $r$ and $M_{0}$ are dimensionless nonvanishing free parameters of the overlap lattice fermion formalism and $U_{\mu}(m)$ is gauge field on links. Other notations are standard. We have introduced a new parameter $t$. The original overlap fermion corresponds to $t=1$. For notational simplicity, we define

$$
A \equiv \frac{1}{a}\left(d r-M_{0}\right), \quad B \equiv \frac{r t}{2 a}, \quad C \equiv \frac{t}{2 a},
$$

and

$$
\begin{aligned}
\Gamma_{\mu}^{-}(m, n) & \equiv \delta_{m+\mu, n} U_{\mu}(m)-\delta_{m, n+\mu} U_{\mu}^{\dagger}(n), \\
\Gamma_{\mu}^{+}(m, n) & \equiv \delta_{m+\mu, n} U_{\mu}(m)+\delta_{m, n+\mu} U_{\mu}^{\dagger}(n)
\end{aligned}
$$

In terms of the above quantities,

$$
\begin{gathered}
X_{m n}=A \delta_{m n}+C \sum \gamma_{\mu} \Gamma_{\mu}^{-}(m, n)-B \sum \Gamma_{\mu}^{+}(m, n), \\
\left(X^{\dagger}\right)_{m n}=A \delta_{m n}-C \sum \gamma_{\mu} \Gamma_{\mu}^{-}(m, n)-B \sum \Gamma_{\mu}^{+}(m, n) .
\end{gathered}
$$

\footnotetext{
${ }^{1}$ As we explained in paper I, the $t$-dependence of the operator $D(m, n)$ is absorbed by a redefinition of $M_{0}$. However introducing the parameter $t$ makes the calculations below quite transparent.
} 
From Eq.(2.3), $B, C=O(t)$ and we consider $A=O(1)$ in the later discussion. Then it is rather straightforward to expand $D(m, n)$ in powers of $t$,

$$
\begin{aligned}
a D(m, n)=2 & \theta(A) \delta_{m n}+\frac{C}{|A|} \sum \gamma_{\mu} \Gamma_{\mu}^{-}(m, n) \\
& +\frac{B C}{2 A|A|} \sum \gamma_{\mu}\left(\Gamma_{\mu}^{-}(m, l) \Gamma_{\nu}^{+}(l, n)+\Gamma_{\nu}^{+}(m, l) \Gamma_{\mu}^{-}(l, n)\right) \\
& +\frac{C^{2}}{2 A|A|} \sum \gamma_{\mu} \gamma_{\nu} \Gamma_{\mu}^{-}(m, l) \Gamma_{\nu}^{-}(l, n)+O\left(t^{3}\right) .
\end{aligned}
$$

Higher-order terms of $t$ are nonlocal and the $t$-expansion corresponds to a kind of the hopping expansion. In the free field case or at the weak gauge coupling, the parameter region in which fermion propagator has no species doublers is easily identified in the $\left(M_{0}, r\right)$ plane. However in the strong-coupling theory like QCD, the parameter region of physical relevance should be determined by other requirements, because the pole in the quark propagator is not a physical observable. Therefore it is important to study the lattice QCD with the overlap fermions in rather wide region of the parameter space.

For the negative $A$ case, which we are interested in later discussion, the leadingorder term of $a D(m, n)$ in Eq.(2.7) is nothing but the naive fermion Dirac operator. As well-known, the naive lattice fermion has larger symmetries than the continuum Dirac fermion. As a result, there appear massless modes other than the pions if the chiral symmetry is spontaneously broken. To avoid this, the terms up to at least $O\left(t^{2}\right)$ in Eq.(2.7) must be taken for practical calculations. This point and its relation to the species doubling will be discussed rather in detail after obtaining the low-energy effective action of hadrons.']

It is verified that the Ginsparg-Wilson (GW) relation [6]

$$
D \gamma_{5}+\gamma_{5} D=a D \gamma_{5} D
$$

\footnotetext{
${ }^{2}$ It has been conjectured that the (weak-coupling) doubling problems might be irrelevant to strong-coupling confining models if the extra symmetries of naive fermions are (explicitly) broken. See for example Ref. [5].
} 
is satisfied by the $t$-expanded $D(m, n)$ in Eq.(2.7) at each order of $t$. Action of the fermion $S_{F}$ in Eq.(2.1) is invariant under the following extended chiral transformation discovered by Lüscher [4],

$$
\delta \psi(m)=\epsilon \gamma_{5}\left(\delta_{n m}-a D(m, n)\right) \psi(n), \quad \delta \bar{\psi}(m)=\epsilon \bar{\psi}(m) \gamma_{5}
$$

where $\epsilon$ is an infinitesimal transformation parameter.

Total action of the lattice QCD is given by

$$
\begin{aligned}
S_{t o t} & =S_{G}+S_{F, M}, \\
S_{G} & =-\frac{1}{g^{2}} \sum_{p l} \operatorname{Tr}\left(U U U^{\dagger} U^{\dagger}\right), \\
S_{F, M} & =S_{F}-M_{B} \sum \bar{\psi}(m) \psi(m),
\end{aligned}
$$

where we have added the bare mass term of quarks. We shall consider the strongcoupling limit in this paper though a systematic strong-coupling expansion is possible. We consider the $U(N)$ gauge group for large $N$. It is easily verified that the following composite operators are covariant under the transformation (2.9) [7, 8],

$$
\bar{q}(n) \equiv \bar{\psi}(n), \quad q(n) \equiv\left(1-\frac{a}{2} D(n, m)\right) \psi(n)
$$

that is

$$
\delta q(m)=\epsilon \gamma_{5} q(m), \quad \delta \bar{q}(m)=\epsilon \bar{q}(m) \gamma_{5}
$$

Hereafter we often set the lattice spacing $a=1$. We consider the case of negative $A$ which is expected to have desired properties of QCD [3].

\section{$3 \quad$ Effective action of hadrons}

Partition function of the $U(N)$ QCD is given by the following functional integral,

$$
Z[J]=\int D \bar{\psi} D \psi D U \exp \left\{-S_{t o t}+\sum J(n) \hat{Q}(n)\right\}
$$


where $[D U]$ is the Haar measure and

$$
\begin{aligned}
& J(n) \hat{Q}(n)=J_{\beta}^{\alpha}(n) \hat{Q}_{\alpha}^{\beta}(n), \\
& \hat{Q}_{\alpha}^{\beta}(n)=\frac{1}{N} \sum_{a} q_{a, \alpha}(n) \bar{q}^{a, \beta}(n),
\end{aligned}
$$

with color index $a$ and spinor-flavor indices $\alpha$ and $\beta$. It should be remarked that the source $J$ is coupled to the nonlocal operator $\hat{Q}$ instead of $\sum_{a} \psi_{a, \alpha}(n) \bar{\psi}^{a, \beta}(n)$. In Ref. 99 we studied the gauged Gross-Neveu model with the overlap fermions and showed that $\hat{Q}_{\alpha}^{\beta}(n)$ is the proper composite fields for the extended chiral symmetry, i.e., the order parameter is given by $\hat{Q}_{\alpha}^{\beta}(n) \delta_{\alpha \beta}$ and the Nambu-Goldstone bosons correspond to $\operatorname{tr}_{S}\left(\hat{Q}(n) \gamma_{5}\right)$ where $\operatorname{tr}_{S}$ is the trace over spinor indices. In the rest of this section, we shall derive the effective action $S_{\text {eff }}(\mathcal{Q})$ defined as

$$
Z[J]=\int D \mathcal{Q} e^{-S_{e f f}(\mathcal{Q})+J \mathcal{Q}}
$$

where integral over color-singlet elementary "meson" field $\mathcal{Q}_{\beta}^{\alpha}$ is defined as in Ref.[3].

In order to evaluate the partition function (3.1), we make a change of variables as

$$
(\bar{\psi}, \psi) \Rightarrow(\bar{q}, q)
$$

Then the measure of the functional integral is transformed as

$$
[d \bar{\psi} d \psi] \Rightarrow[d \bar{q} d q] e^{N \operatorname{Tr} \ln \left(1-\frac{a}{2} D\right)}
$$

where $\operatorname{Tr}$ in (3.4) is the trace over the spinor-flavor as well as the real-space indices. The contribution from the Jacobian $\operatorname{Tr} \ln \left(1-\frac{a}{2} D\right)$ is easily evaluated by the t-expansion. In terms of $\bar{q}$ and $q$,

$$
\begin{aligned}
S_{F}^{\prime}(q)= & S_{F}(\psi) \\
= & \bar{q}(m)\left[\frac{C}{|A|} \sum \gamma_{\mu} \Gamma_{\mu}^{-}(m, n)+\frac{B C}{2 A|A|} \sum \gamma_{\mu}\left(\Gamma_{\mu}^{-}(m, l) \Gamma_{\nu}^{+}(l, n)+\Gamma_{\nu}^{+}(m, l) \Gamma_{\mu}^{-}(l, n)\right)\right. \\
& \left.+O\left(t^{3}\right)\right] q(n) .
\end{aligned}
$$


The term of $O\left((C / A)^{2}\right)$ in $(2.7)$ has disappeared. As explained in paper I, this term is exactly determined from the term of $O(C / A)$ in $(2.7)$ through the GW relation. We expect that a similar phenomenon occurs for higher-order terms of the $t$-expansion which are determined by lower-order terms through the GW relation. That is, the terms of higher-order of $t$ which are determined through the GW relation drop when the action is rewritten in terms of $q$ and $\bar{q}$. The mass term is also given by

$$
\begin{aligned}
M_{B} \bar{\psi}(m) \psi(m)= & M_{B} \bar{q}(m)\left[\delta_{m n}-\frac{C}{2 A} \sum \gamma_{\mu} \Gamma_{\mu}^{-}(m, n)\right. \\
& -\frac{B C}{4 A^{2}} \sum \gamma_{\mu}\left(\Gamma_{\mu}^{-}(m, l) \Gamma_{\nu}^{+}(l, n)+\Gamma_{\nu}^{+}(m, l) \Gamma_{\mu}^{-}(l, n)\right) \\
& \left.+O\left(t^{3}\right)\right] q(n) .
\end{aligned}
$$

Total action of $q$ is then given by

$$
S_{F, M}^{\prime}(q)=S_{F, M}(\psi)
$$

Integral over the gauge field can be performed by the one-link integral,

$$
e^{W(\bar{D}, D)}=\int d U_{\mu} \exp \left[\operatorname{Tr}\left(\bar{D}_{\mu} U_{\mu}+U_{\mu}^{\dagger} D_{\mu}\right)\right]
$$

where $D_{\mu}$ and $\bar{D}_{\mu}$ are bilinears of Grassmann numbers. Explicit form of the one-link integral $W(\bar{D}, D)$ for the present system is obtained as in paper I. Gauge fields in $\Gamma_{\mu}^{ \pm}(m, n)$ are replaced with composite operators of $q$ and $\bar{q}$ after the integral over $U_{\mu}(n)$. (For details see paper I.) After some calculation,

$$
\int D U e^{-S_{F, M}^{\prime}(q)+N \operatorname{Tr} \ln \left(1-\frac{1}{2} D\right)+\sum J \hat{Q}}=e^{-N M_{B} \operatorname{tr}(\hat{Q})-S_{2}(\hat{Q})+\sum J \hat{Q}}
$$

where $\operatorname{tr}$ in $(3.9)$ is the trace over spinor-flavor indices and $S_{2}(\hat{Q})$ is some complicated function of $\hat{Q}$ which is obtained in powers of $t$. Let us define the following operators;

$$
\begin{aligned}
\epsilon & =\epsilon_{\mu}^{\delta \sigma}(m)=\left(\frac{C}{A}\right)^{2}\left(1-\frac{M_{B}}{2}\right)^{2}\left(\hat{Q}(m) \gamma_{\mu} \hat{Q}(m+\mu) \gamma_{\mu}\right)^{\delta \sigma} \\
\epsilon^{\prime} & =\epsilon_{\mu}^{\prime \delta \sigma}(m)=\left(\frac{C}{A}\right)^{2}\left(1-\frac{M_{B}}{2}\right)^{2}\left(\hat{Q}(m+\mu) \gamma_{\mu} \hat{Q}(m) \gamma_{\mu}\right)^{\delta \sigma}
\end{aligned}
$$


Then in terms of $\epsilon^{\prime} \mathrm{s}, S_{2}(\hat{Q})$ is given as

$$
\begin{aligned}
\frac{1}{N} S_{2}(\hat{Q})= & -\sum_{m, \mu} \operatorname{tr}\left[g\left(\epsilon_{\mu}(m)\right)\right] \\
& +\frac{B C^{3}}{2 A^{4}}\left(1-\frac{M_{B}}{2}\right)^{3} \\
& \times \sum_{m, \mu, \nu}\left\{\operatorname{tr}\left[\mathcal{Q}(m+\mu) \gamma_{\mu} g^{\prime}\left(\epsilon_{\mu}(m)\right) \mathcal{Q}(m) \gamma_{\mu} \mathcal{Q}(m+\mu+\nu) \gamma_{\nu} g^{\prime}\left(\epsilon_{\nu}(m+\mu)\right)\right]\right. \\
& -\operatorname{tr}\left[\mathcal{Q}(m+\mu+\nu) \gamma_{\mu} g^{\prime}\left(\epsilon_{\mu}(m+\nu)\right) \mathcal{Q}(m+\nu) \gamma_{\mu} \mathcal{Q}(m+\mu) \gamma_{\nu} g^{\prime}\left(\epsilon_{\nu}^{\prime}(m+\mu)\right)\right] \\
& +\operatorname{tr}\left[\mathcal{Q}(m) \gamma_{\mu} g^{\prime}\left(\epsilon_{\mu}^{\prime}(m)\right) \mathcal{Q}(m+\mu) \gamma_{\mu} \mathcal{Q}(m+\nu) \gamma_{\nu} g^{\prime}\left(\epsilon_{\nu}(m)\right)\right] \\
& -\operatorname{tr}\left[\mathcal{Q}(m+\nu) \gamma_{\mu} g^{\prime}\left(\epsilon_{\mu}^{\prime}(m+\nu)\right) \mathcal{Q}(m+\mu+\nu) \gamma_{\mu} \mathcal{Q}(m) \gamma_{\nu} g^{\prime}\left(\epsilon_{\nu}^{\prime}(m)\right)\right] \\
& +\operatorname{tr}\left[\mathcal{Q}(m+\nu) \gamma_{\nu} g^{\prime}\left(\epsilon_{\nu}(m)\right) \mathcal{Q}(m) \gamma_{\mu} \mathcal{Q}(m+\mu+\nu) \gamma_{\mu} g^{\prime}\left(\epsilon_{\mu}(m+\nu)\right)\right] \\
& +\operatorname{tr}\left[\mathcal{Q}(m+\mu+\nu) \gamma_{\nu} g^{\prime}\left(\epsilon_{\nu}(m+\mu)\right) \mathcal{Q}(m+\mu) \gamma_{\mu} \mathcal{Q}(m+\nu) \gamma_{\mu} g^{\prime}\left(\epsilon_{\mu}^{\prime}(m+\nu)\right)\right] \\
& -\operatorname{tr}\left[\mathcal{Q}(m) \gamma_{\nu} g^{\prime}\left(\epsilon_{\nu}^{\prime}(m)\right) \mathcal{Q}(m+\nu) \gamma_{\mu} \mathcal{Q}(m+\mu) \gamma_{\mu} g^{\prime}\left(\epsilon_{\mu}(m)\right)\right] \\
& \left.-\operatorname{tr}\left[\mathcal{Q}(m+\mu) \gamma_{\nu} g^{\prime}\left(\epsilon_{\nu}^{\prime}(m+\mu)\right) \mathcal{Q}(m+\mu+\nu) \gamma_{\mu} \mathcal{Q}(m) \gamma_{\mu} g^{\prime}\left(\epsilon_{\mu}^{\prime}(m)\right)\right]\right\} \\
& +\frac{N_{s f} C^{4}}{4 A^{4}}\left(1-\frac{M_{B}}{2}\right)^{2} \sum_{m, \mu} \operatorname{tr}\left[\mathcal{Q}(m+\mu) \gamma_{\mu} g^{\prime}\left(\epsilon_{\mu}(m)\right) \mathcal{Q}(m) \gamma_{\mu} g^{\prime}\left(\epsilon_{\mu}^{\prime}(m)\right)\right] \\
& +O\left(t^{3}\right),
\end{aligned}
$$

where $N_{s f}$ is the dimension of the spinor-flavor index and

$$
g(x)=1-(1-4 x)^{\frac{1}{2}}+\ln \left[\frac{1}{2}\left(1+(1-4 x)^{\frac{1}{2}}\right)\right] .
$$

Elementary meson fields $\mathcal{Q}$ and their functional integral are introduced as in the previous case [3, 10],

$$
\begin{aligned}
\int d \bar{q} d q \exp \left(\frac{1}{N} J_{\alpha}^{\beta} q_{a}^{\alpha} \bar{q}_{\beta}^{a}\right) & =(\operatorname{det} J)^{N} \\
& =\oint d \mathcal{Q}(\operatorname{det} \mathcal{Q})^{-N} \cdot e^{J \cdot \mathcal{Q}}
\end{aligned}
$$

where the integral over $\mathcal{Q}$ is defined by the contour integral, i.e., $\mathcal{Q}$ is polar-decomposed as $\mathcal{Q}=R V$ with positive-definite Hermitian matrix $R$ and unitary matrix $V$, and

\footnotetext{
${ }^{3}$ As we shall see, $\mathcal{Q} \sim O\left(t^{-1}\right)$.
} 
$\oint d \mathcal{Q} \equiv \int d V$ with the Haar measure of $\mathrm{U}\left(N_{s f}\right)$ [10. From (3.13), there appear additional terms like $(N \operatorname{Tr} \log \mathcal{Q})$ in the effective action. Therefore the effective action is given by

$$
S_{\text {eff }}(\mathcal{Q})=N \sum_{n}\left[\operatorname{tr} \ln \mathcal{Q}(n)+M_{B} \operatorname{tr} \mathcal{Q}(n)\right]+S_{2}(\mathcal{Q}) .
$$

Effective potential of the chiral condensate is obtained from $S_{\text {eff }}$ in Eq.(3.14) by setting

$$
Q^{\alpha \beta}(n)=v \delta_{\alpha \beta}
$$

In paper I we obtained

$$
v=\frac{|A|}{2 C} \sqrt{\frac{2 d-1}{d^{2}}}+O\left(t^{0}\right)+O\left(M_{B}\right) .
$$

From the above result, we can expect that there appear quasi-Nambu-Goldstone bosons, i.e., pions. Actually we found that there are massless modes in the channel $\operatorname{tr}_{S}\left(\hat{Q} \gamma_{5}\right)=\operatorname{tr}_{S}\left(q \bar{q} \gamma_{5}\right)$ where $\operatorname{tr}_{S}$ is the trace over spinor index. It is straightforward to obtain the effective action of the pions by inserting the following expression of $\mathcal{Q}$ into $S_{\text {eff }}$ in Eq.(3.14),

$$
\mathcal{Q}(m)=v e^{i \gamma_{5} \phi_{5}(m)} .
$$

For example from (3.10) and (3.16), one can easily obtain

$$
\epsilon_{\mu} \propto e^{-i \gamma_{5} \nabla_{\mu} \phi_{5}}
$$

After some calculation, we have

$$
\left.S_{e f f}\right|_{\mathcal{Q}(m)=v e^{i \gamma_{5} \phi_{5}(m)}}=2^{\frac{d}{2}} N\left[C_{\pi} \sum_{m, \mu} \operatorname{tr}_{F}\left(\nabla_{\mu} \phi_{5}(m)\right)^{2}-\frac{M_{B} v}{2} \sum_{m} \operatorname{tr}_{F}\left(\phi_{5}(m)\right)^{2}\right],
$$

where $C_{\pi}$ is some positive constant and $\operatorname{tr}_{F}$ is the trace over flavor index. Therefore the fields $\phi_{5}$ are quasi-Nambu-Goldstone pions as expected.

One may think that there appear additional massless excitations in other modes of $\mathcal{Q}(m)$ as in the naive lattice fermion case [10. In the following section we shall argue that this is not the case. There relationship between the species doubling and the symmetries of the lattice fermion becomes clear. 


\section{Symmetries and species doubling}

In paper I we showed that Lüscher's chiral symmetry is spontaneously broken at strong coupling. In the previous section, we derived the effective action and showed that pions appear in the desired form. As well-known, the naive fermion has much larger symmetry than the continuum fermion, and as a result there appear massless vector boson, etc. besides pions. In this section, we shall examine symmetries of the $t$-expanded overlap fermions and argue that such extra "Nambu-Goldstone bosons" do not appear because of the higher-order terms of $t$. In that discussion, we show that these extra (quasi-)massless bosons are nothing but pion like bound state of quark and its doublers. Finally we shall discuss physical meaning of the hopping expansion especially in the confining phase.

The naive lattice fermion action has "spectrum doubling symmetry", that is, the action is invariant under the following transformation,

$$
\begin{aligned}
\psi(n) \rightarrow & e^{-i n \cdot \pi_{H}} M_{H} \psi(n), \\
\bar{\psi}(n) \rightarrow \quad & \bar{\psi}(n) M_{H}^{\dagger} e^{i n \cdot \pi_{H}}, \\
\text { where } \quad & n \cdot \pi_{H}=\sum_{\mu=1}^{d} n_{\mu} \pi_{H, \mu}, \\
H= & \left\{\mu_{1}, \cdots, \mu_{h}\right\}, \quad\left(\mu_{1}<\mu_{2}<\cdots<\mu_{h}, 1 \leq h \leq d\right), \\
\text { explicitly } \quad\{\mu\}=\{1\}, \cdots,\{d\},\left\{\mu_{1}, \mu_{2}\right\}=\{1,2\},\{2,3\},\{1,3\}, \cdots, & \left\{\mu_{1}, \mu_{2}, \cdots, \mu_{d}\right\}=\{1,2, \cdots, d\}, \\
M_{H}= & M_{\mu_{1}} M_{\mu_{2}} \cdots M_{\mu_{h}}, \\
M_{\mu}= & i \gamma_{5} \gamma_{\mu}, \\
\pi_{H, \mu}= & \left\{\begin{array}{c}
\pi \text { if } \mu \in H \\
0 \quad \text { otherwise. }
\end{array}\right.
\end{aligned}
$$

For example, under the transformation $H=\{\mu\}$, right(left)-handed fermion at momentum $p=0$ transforms to left(right)-handed fermion at $p=\left(0, \ldots, p_{\mu}=\pi, 0, \ldots\right)$. Furthermore we can diagonalize the spinor indices of fermions by rewriting the naive- 
fermion action $S_{\text {naive }}$ in terms of the following variables $\chi_{n}$ and $\bar{\chi}_{n}$,

$$
\begin{aligned}
& \psi(n)=A_{n} \chi_{n}, \\
& \bar{\psi}(n)=\bar{\chi}_{n} A_{n}^{\dagger}, \\
& A_{n}=\gamma_{1}^{n_{1}} \gamma_{2}^{n_{2}} \cdots \gamma_{d}^{n_{d}}, \\
& A_{n}^{\dagger} \gamma_{\mu} A_{n \pm \hat{\mu}}=(-1)^{n_{1}+\cdots n_{\mu-1}} 1, \\
& S_{\text {naive }}=\sum \bar{\chi}_{m} \eta_{\mu}(n)\left[\delta_{m+\mu, n} U_{\mu}(m)-\delta_{m, n+\mu} U_{\mu}^{\dagger}(n)\right] \chi_{n},
\end{aligned}
$$

where $\eta_{\mu}(n)=\frac{1}{2}(-)^{n_{1}+\cdots+n_{\mu-1}}$ and we have set the lattice spacing $a=1$.

As spinor indices of $\chi_{n}$ and $\bar{\chi}_{n}$ are diagonal in (4.4), the naive fermion action has global $U\left(N_{s f}\right) \otimes U\left(N_{s f}\right)$ symmetry which is manifest in the $\chi$-representation [10]. By the chiral condensation $\left\langle\bar{\chi}_{n} \chi_{n}\right\rangle \neq 0$, the $U\left(N_{s f}\right) \otimes U\left(N_{s f}\right)$ symmetry is spontaneously broken down to $U\left(N_{s f}\right)$. On the other hand, one can easily see how the fields $\chi_{n}$ and $\bar{\chi}_{n}$ transform under (4.1). For example, for $H=\{\mu\}$,

$$
\begin{aligned}
& \chi_{n} \rightarrow i \gamma_{5} \gamma_{\mu} \chi_{n}, \\
& \bar{\chi}_{n} \rightarrow \bar{\chi}_{n} i \gamma_{5} \gamma_{\mu},
\end{aligned}
$$

and similar expression for other doubling transformation $H$. Then it is obvious that the doubling symmetry (4.1) is a part of the $U\left(N_{s f}\right)$ symmetry. This indicates that the extra Nambu-Goldstone bosons are nothing but pion like bound state of the original quark and its doublers (for more details, see discussion below). By practical calculation, we can verify that higher-order terms of $t$ in Eq.(2.7) explicitly breaks the $U\left(N_{s f}\right) \otimes U\left(N_{s f}\right)$ symmetry down to the flavour symmetry $U\left(N_{f}\right)$ as desired. This is due to the Wilson term $B(t ; m, n)$ in Eq.(2.2).

Next let us study the doubling spectrum of pions, e.g., "pions" which are composed of quark $q(p \sim 0)$ and, e.g., anti-quark $\bar{q}\left(p_{\mu} \sim \pi, p_{\nu \neq \mu} \sim 0\right)$ 用. From the doubling

\footnotetext{
${ }^{4}$ As quarks are confined, concept of "quark with vanishing momentum" etc does not have any strict meaning. However, we hope that what we are meaning in the present context is quite clear for readers.
} 
transformation (4.1), we should consider the following composite fields,

$$
\hat{Q}^{\prime}(n) \equiv \frac{1}{N} \sum q(n) \bar{q}(n) M_{H}^{\dagger} e^{i n \cdot \pi_{H}}
$$

For example for $H=\{\mu\}$,

$$
\hat{Q}^{\prime}=\hat{Q} i \gamma_{\mu} \gamma_{5}(-)^{n_{\mu}}
$$

From the above consideration, the bound state of quark $q(p \sim 0)$ and anti-quark $\bar{q}\left(p_{\mu} \sim \pi, p_{\nu \neq \mu} \sim 0\right)$ is described by the "vector" field $\phi_{\mu}$ introduced as follows,

$$
Q=v e^{i \gamma_{\mu} \phi_{\mu}(-1)^{n_{\mu}}}
$$

where the flavour index is arbitrary.

It is rather straightforward to obtain the effective action of $\phi_{\mu}$ by substituting Eq.(4.8) into the effective action $S_{\text {eff }}(\mathcal{Q})$ and using the formulae like

$$
\begin{aligned}
\epsilon_{\mu}(m) & =\left(\frac{C}{A}\right)^{2}\left(1-\frac{M_{B}}{2}\right)^{2} v^{2} e^{-i(-1)^{m_{\nu}} \gamma_{\nu} \nabla_{\mu} \phi_{\nu}(m)} \\
\epsilon_{\mu}(m)^{\prime} & =\left(\frac{C}{A}\right)^{2}\left(1-\frac{M_{B}}{2}\right)^{2} v^{2} e^{i(-1)^{m_{\nu}} \gamma_{\nu} \nabla_{\mu} \phi_{\nu}(m)}
\end{aligned}
$$

It is verified that at the leading order of $t$, i.e., the naive fermion, $\phi_{\mu}$ has a mass like (mass $)^{2} \propto M_{B}$ just as the pions $\phi_{5}$. At $O\left(t^{2}\right)$, no mass terms appear (accidentally). We therefore examine the terms of $O\left(t^{3}\right)$ of the hopping expansion like

$$
\frac{B^{2} C}{A^{3}} \sum \gamma_{\mu} \Gamma_{\mu}^{-}(m, l) \Gamma_{\nu}^{+}(l, v) \Gamma_{\alpha}^{+}(v, n)+\cdots
$$

The terms in Eq.(4.11) generate the following terms in the effective action $S_{\text {eff }}(\mathcal{Q})$,

$$
\begin{aligned}
& \sum_{m, \mu, \nu, \alpha} \operatorname{tr}\left[\mathcal{Q}(m) \gamma_{\mu} \mathcal{Q}(m+\mu+\nu+\alpha) \gamma_{\alpha} g^{\prime}\left(\epsilon_{\alpha}(m+\mu+\nu)\right) \mathcal{Q}(m+\mu+\nu)\right. \\
& \left.\times \gamma_{\nu} g^{\prime}\left(\epsilon_{\nu}(m+\mu)\right) \mathcal{Q}(m+\mu) \gamma_{\mu} g^{\prime}\left(\epsilon_{\mu}(m)\right)\right]+\cdots
\end{aligned}
$$

By substituting the expression (4.8) into Eq.(4.12), one can verify that the mass term of $\phi_{\mu}$, i.e., the term like $\phi_{\mu}^{2}$, actually appears at $O\left(t^{3}\right)$. 
Similar consideration is straightforward for other bound state of quark and its doublers. These excitations are described by the following modes,

$$
Q=v e^{i \gamma_{\mu} \gamma_{\nu} \gamma_{5} \phi_{\mu \nu}(-)^{n_{\mu}+n_{\nu}}}
$$

etc. We expect that they all acquire a finite mass at higher order of $t$ as the symmetry of the action indicates. If the above expectation is correct, the result implies that the doublers at weak coupling do not produce any ill effect in the spectrum of light mesons at strong coupling except the $U(1)$ problem, though the result is obtained by using the $t$-expansion in the above study. Recently, Golterman and Shamir gave careful study on QCD with the overlap fermions with small hopping parameter and they obtained the result which supports the above argument [11]. Relationship between the $U(1)$ problem and the $t$-expansion and/or the strong-coupling expansion will be briefly discussed in Sect.5.

So far we have considered bound state of pairs like quark $q(p \sim 0)$ and antiquark $\bar{q}\left(p_{\mu} \sim \pi\right)$ and found that they are massive. In the rest of this section, we shall reexamine the pions $\phi_{5}$ more precisely. Pions $\phi_{5}$ consist of the bound state of quark $q(p \sim 0)$ and anti-quark $\bar{q}(p \sim 0)$. However besides that component, there are contributions from other pairs of quark and anti-quark like $q\left(p_{\mu} \sim \pi\right)$ and $\bar{q}\left(p_{\mu} \sim \pi\right)$, i.e., bound state of doublers with the same momentum, i.e.,

$$
\phi_{5} \sim \bar{q}(p \sim 0) \gamma_{5} q(p \sim 0)+\lambda \cdot \bar{q}\left(p_{\mu} \sim \pi\right) \gamma_{5} q\left(p_{\mu} \sim \pi\right)+\cdots
$$

It is interesting to estimate the weight of these bound pairs $\lambda$.

To this end, let us first consider the weak-coupling region or small lattice spacing and $U_{\mu}(m) \sim 1$. From $S_{F}^{\prime}(q)$ in (3.5), the free propagator of $q$ is obtained from the following expression of the $t$-expanded action,

$$
S_{F}^{\prime}(q) \sim \bar{q}(p)\left[\frac{C}{|A|} \sum \gamma_{\mu} i \sin p_{\mu}+\frac{B C}{A|A|} \sum \gamma_{\mu} i \sin p_{\mu} \cos p_{\nu}\right] q(p)+\cdots
$$

where we have ignored coefficients which are irrelevant for the discussion. From 
Eq.(4.15), for quark with momentum $p \sim 0$,

$$
S_{F}^{\prime}(q) \sim \bar{q}(p)\left(\frac{C}{|A|}+\frac{B C d}{A|A|}\right) \sum\left(i \gamma_{\mu} p_{\mu}\right) q(p)
$$

whereas for doubler at $p_{\nu} \sim \pi$ the second term in (4.15) changes sign because of the factor $\cos p_{\nu}$, and therefore

$$
S_{F}^{\prime}(q) \sim \bar{q}(p)\left(\frac{C}{|A|}+\frac{B C(d-2)}{A|A|}\right) \sum\left(i \gamma_{\mu} p_{\mu}\right) q(p),
$$

with the suitable redefinition of the momentum $p_{\nu} \rightarrow \pi-p_{\nu}$. This means that at the leading order of the hopping expansion the doublers have the same weight or residue with the original fermion at $p \sim 0$. However at $O\left(t^{2}\right)$, residue of doublers is reduced though the fermion propagator has a pole at the doubler momentum. That is, in the hopping expansion (for the free field case), the doublers appear as a genuine particle but their probability is decreased by the higher-order terms of $t$. On the other hand, exact form of the free fermion propagator is obtained readily. Physical parameter region of the overlap fermions is identified by requiring that the propagator has a pole only at $p=0$ and no poles at $p=\pi$. In this sense, the $t$-expansion is not a good approximation in the perturbative deconfinement phase. However in the parameter region in which the $t$-expansion converges, we can see that doublers are suppressed in the overlap formalism.

The above discussion can be easily extended for smooth gauge field configurations or small gauge coupling $g$. In this case, there appears an additional term proportional to the gauge potential $i g A_{\mu}=\ln U_{\mu}$ which is common to fermion with small momentum and its doublers. That is, both in Eqs.4.16) and (4.17),

$$
\bar{q}(p)\left(\gamma_{\mu} p_{\mu}\right) q(p) \rightarrow \bar{q}(k) \gamma_{\mu}\left(\delta(k-p) p_{\mu}-g A_{\mu}(k-p)\right) q(p)
$$

Then weights of doublers are suppressed by the term of $O\left(t^{2}\right)$ for smooth $A_{\mu}$ as in the free case.

\footnotetext{
${ }^{5}$ Here we assume negative $A$ as in the previous discussions.
} 
In the confining phase on the other hand, poles in the quark propagator are not physical observables. We expect that the $t$-expansion gives at least qualitatively correct picture for that phase. The above consideration for the weak-coupling case suggests that the weight $\lambda$ in Eq.(4.14) is a decreasing function of the hopping parameter $t$ for the probability of doublers decreases as $t$ increases. As $t$ increases further, convergence of the expansion breaks down. We think that this nonconvergence of the $t$-expansion is closely related with the $U(1)$ problem. This point will be discussed in the following section. At the strong-coupling limit, the convergence of the $t$-expansion is improved and also the discrimination between "quark with small momentum" and its doublers becomes obscure because of the large fluctuation of the gauge field $U_{\mu}(n)$. In order to clarify the phase structure of the QCD with overlap fermions, numerical studies are required. We hope that the studies by the $t$-expansion will be useful for further studies on that problem.

\section{Discussion}

In paper I, we developed the hopping expansion for the overlap fermions and showed that Lüscher's chiral symmetry is spontaneously broken at strong gauge coupling. In this paper, we derived the effective action of mesons and showed that pions appear in the desired form. It was also argued that other extra "Nambu-Goldstone" bosons do not appear because of the explicit breaking of the $U\left(N_{s f}\right) \otimes U\left(N_{s f}\right)$ symmetry $\rightarrow U\left(N_{f}\right)$ which exists in the naive lattice fermion.

Important problem is whether the $t$-expansion covers physically relevant parameter region of the lattice QCD. It is not straightfoward to answer this question because poles in the quark propagator is not physical observable in the confinement phase. However effect of the chiral anomaly must be appear in the physical parameter region. In QCD, the $U(1)$ problem is a suitable object to identify the correct parameter region. We expect that a mass term of the flavour singlet meson will appear from the 
Jacobian in (3.4). This is a solution to the $U(1)$ problem. However if this Jacobian $\operatorname{Tr} \ln \left(1-\frac{1}{2} D\right)$ and the action itself are both expandable in powers of $t$, in other words, local in the real space in a broad sense, the total action $S_{F, M}^{\prime}(q)-N \operatorname{Tr} \ln \left(1-\frac{1}{2} D\right)$ with $M_{B}=0$ is obviously invariant under the transformation (2.12). Therefore flavour singlet Nambu-Goldstone boson also appears as a result of the spontaneous breaking of (2.12). Recently careful study on the overlap-Dirac fermions with a small hopping parameter is given [11]. There the same result with above is obtained.

We expect that convergence of the $t$-expansion breaks down first in the Jacobian because it contains (infinitely) higher-power terms of the operator $D$. Then a wouldbe Nambu-Goldstone boson becomes massive because of the nonlocality' Problem is whether this nonconvergence of the Jacobian factor means a phase transition from small to intermediate (physical) value of $t$. If this is a phase transition, results of the present study may be useless for investigation on the physical properties of the intermediate- $t$ phase. Of course it depends on nature of the phase transition. This problem is under study and we hope that result will be reported in a future publication [12].

Finally let us notice recent studies on the domain wall fermions at strong coupling. Very recently lattice $U(1)$ gauge model with the domain-wall fermions was studied in the strong-coupling limit by the Hamiltonian formalism [13]. There an effective Hamiltonian for low-lying color-singlet degrees of freedom is obtained by treating the terms proportional to the gauge fields $U_{\mu}(m)$ as perturbations. This idea is very close to ours in paper I and the present paper though we employ the Lagrangian formalism. It is very interesting and also useful to investigate the relation between the results obtained in Ref. 13] and ours in detail. This may give an important insights to numerical studies on QCD with the domain-wall and /or overlap fermions.

\footnotetext{
${ }^{6}$ Under the chiral transformation (2.12), the operator $D$ is invariant. However after integration over the gauge field, the Jacobian is expressed in terms of $q$. Then it is possible that symmetrybreaking terms appear there. In other words, the transformation from $\psi$ to $q$ becomes singular above a certain critical value of $t_{C}$.
} 


\section{Acknowledgements}

KN, one of the authors, would like to thank Prof.T.Yoneya for useful discussions.

We also thank the referee of this paper for pointing out the references in Ref. [5]. 


\section{References}

[1] R.Narayanan and H.Neuberger, Nucl.Phys. B412(1994)574;

Nucl.Phys.B443(1995)305.

[2] H.Neuberger, Phys.Lett.B417(1998)141.

[3] I.Ichinose and K.Nagao, Nucl.Phys.B577(2000)279.

[4] M.Lüscher, Phys.Lett.B428(1998)342.

[5] B.Svetitsky, S.D.Drell, H.R.Quinn and M.Weinstein, Phys.Rev.D22(1980)490;

M.Weinstein, S.D.Drell, H.R.Quinn and B.Svetitsky, Phys.Rev.D22(1980)1190;

K.Melnikov and M.Weinstein, hep-lat/0004016.

[6] P.H.Ginsparg and K.G.Wilson, Phys.Rev.D25(1982)2649.

[7] F.Niedermayer, Nucl.Phys.Proc.Suppl.73(1999)105.

[8] Y.Kikukawa and T.Noguchi, het-lat/9902022.

[9] I.Ichinose and K.Nagao, Mod.Phys.Lett.A15(2000)857.

[10] N.Kawamoto and J.Smit, Nucl.Phys.B192(1981)100.

[11] M.Golterman and Y.Shamir, JHEP 0009(2000)006.

[12] I.Ichinose and K.Nagao, work in progress.

[13] R.C.Brower and B.Svetitsky, Phys.Rev.D61(2000)114511. 